\title{
Prevalence and triggering factors of unintended pregnancies among women in India: Evidence from Indian Demographic and Health Survey 2015-2016
}

\author{
Md Akhtarul Islam ${ }^{\text {a, * }}$, Abdur Rahim ${ }^{\text {a,1 }}$, Abdul Jabbar ${ }^{\text {b }}$ \\ ${ }^{a}$ Statistics Discipline, Science, Engineering and Technology (SET) School, Khulna University, Khulna, 9208, Bangladesh \\ ${ }^{\mathrm{b}}$ Department of Clinical Medicine, Faculty of Veterinary Science, University of Veterinary and Animal Sciences, Lahore, Punjab, Pakistan
}

\section{A R T I C L E I N F O}

\section{Keywords:}

Women

Unintended pregnancy

Multinomial logit

Determinants

India

\begin{abstract}
A B S T R A C T
Objectives: The primary focus of this study was to estimate the prevalence and assess the associated risk factors that impact unintended pregnancies among women who were married in India. Study design: This study utilized a cross sectional study design.

Methods: It was a cross-sectional study on 32224 pregnant women that was extracted from the most recent Demographic and Health Study organized from 2015 to 2016 (round 7) in India. Facts and statistics were examined by utilizing different data analysis techniques, i.e., univariate, bivariate and multivariate analysis.

Results: Nearly $11.9 \%$ of cases were unplanned pregnancies, out of which $7 \%$ were early pregnancies. The mistimed pregnancy for women aged 15-19 years was $7.896(6.246,9.981)$ times more likely than the women of age 30 . The women living in urban areas were having $0.877(0.778,0.988)$ times fewer chances to have an early parturiency as compared to those living in rural areas. Women who got married before 18 years of age were $0.583(0.524,0.650)$ times less likely to have mistimed pregnancy than the women who got married at the age of 18 and over. Women with no children were $0.217(0.176,0.268)$ times less likely to have early pregnancy than women with more than three children.

Conclusion: Factors that enhance the probability of unfavorable pregnancies are the age at first marriage, number of dead children, wealth status, fertility preference, and family planning knowledge. This survey suggests strengthening the strategies of family planning so that the ratio of unplanned pregnancies decreases.
\end{abstract}

1. What we already know

> Approximately 85 million women face unintended pregnancies every year worldwide. Consequently, unsafe abortions and maternal deaths are very high.

$>$ Research from India suggested that Lower utilization of maternal health facilities and poorer outcomes of infant health are influenced by unintended pregnancy

- Enriched research findings showed that the reason and association of unintended pregnancy in India is scarce.

\section{What this article adds}

$>$ In this study, significant factors were found that were affecting unintended pregnancies in India by using the most recent Indian Demographic and Health Survey 2015-2016 data.

> Age of respondent, type of residence, age at first marriage, wealth status, children ever born, and fertility preference were the strongest predictors for unintended pregnancies in India.

$>$ This study illustrates the importance of family planning services and recommends different interventions to reduce the level of unintended pregnancies to achieve the Sustainable Development Goal of reducing maternal deaths.

\footnotetext{
* Corresponding author. Statistics Discipline, Khulna University, Khulna, 9208, Bangladesh.

E-mail address: akhtarulstat@ku.ac.bd (M.A. Islam).

1 The first author and last author thankfully acknowledge the contribution of Late Abdur Rahim for developing the methodology and analysis part of this article. Abdur Rahim's name is kept in the authors' list with the consent of his family member.
} 


\section{Introduction}

To understand fertility, demographers are interested to know the concept of unintended pregnancy, whereas the public health practitioners are intended to prevent unwanted childbearing, that is relying on the concept of unintended pregnancy. ${ }^{1}$ Most married women having low income have the most significant difficulty taking responsibility for an unintended child. ${ }^{2}$ There are several adverse effects of unintended pregnancy on mental health, antenatal care, postnatal care, curative care, breastfeeding, child immunization, and infant mortality. ${ }^{3,4}$ Strong evidence shows that women with an unintended pregnancy have less probability of utilizing these health care services. ${ }^{5,6}$ Unintended pregnancy complicates abortion-related morbidity and mortality, especially in some countries where abortion is not allowed legally. ${ }^{7}$ Every day around one million conceptions occur because of 100 million acts of sexual intercourse. About $50 \%$ of which are unplanned, and about $25 \%$ of pregnancies are unwanted. ${ }^{8}$ This increase in the frequency of unplanned pregnancies can be seen as a marker of women's autonomy for having children's decision-making process. ${ }^{9}$ Though it is a common worldwide phenomenon, minimal published literature focuses on unintended pregnancy determinants in developing countries like India. ${ }^{10}$ Several studies showed that about $50 \%$ of all pregnancies in the USA, $23 \%$ in Iran, $40 \%$ in Nepal, $41 \%$ in Koshu, Japan are unintended. ${ }^{11-13}$ Unintended pregnancies are widespread and can be due to various causes. Contraception failure due to inconsistent or incomplete use of contraceptive pills, failure of family planning to prevent pregnancies, less commonly, and rape are the main reasons behind unintended pregnancy. ${ }^{14,15}$ In the United States, approximately half of the pregnancies are unintended due to contraceptive failure. Amng those unintended pregnancies, almost half of the pregnencies end in induced abortions. $^{16}$

Therefore, unplanned pregnancy is a problem that cannot be neglected. To find out the actual reasons behind unintended pregnancy, the prevalence and causal factors of unplanned pregnancy in India were focused.

\section{Methods}

\subsection{Data sources}

In this study, the most recent Demographic and Health Survey was done from 2015 to 2016 (round 7) in India. The survey was performed in all seven union territories and twenty-nine states of India for the very first time in the national family health survey-4. In India, the assessment of many measures has been permitted at the district level for all six hundred and forty districts according to the 2011 survey. ${ }^{17} \mathrm{~A}$ probability-based cluster sampling technique was used to select survey respondents. The complete database was accessible as per demand from https://dhsprogram.com/, and such database did not comprise any noticeable information on the review members. Demographic and health survey was familiar for its uniformity as well as for nationwide illustrating selection of candidates, objective calculation of anthropometry, gathering different types of surveying and affected assessment markers for fitness and nourishment, and rapid reaction. ${ }^{17}$

32428 out of 699686 reproductive age interviewed (4.6\%) women were at the state of parturiency at the time of the study. Data of 204 females were removed due to lost information. Ultimately, the sample size became32224.

\subsection{Variables}

This dependent variable analysis was parturiency status, determined by respondents' intuit wish for present pregnancy. Women were questioned: "At what time you were pregnant, did you need to get pregnant at that point, did you need to hold up till afterward, or did you not need to have more children at all?" The three acceptable alternatives were needed at that point (wanted), where the pregnancy was required to occur afterward (misplanned) and did not need at all (unfavorable).

The independent variables were chosen in the examination on the basis of their importance in former pregnancy intention status surveys. Some variables were recategorized from their actual groups in the database to make analysis easier and more purposeful. The age of the respondents was categorized into four categories (i.e., age group between 15 and 19,20-24, 25-29, 30, and above). There were two categories in the residence of respondents like urban residents and rural residents. Age at marriage was divided into two categories named married before 18 years and married at 18 years or above age. The husband's residence was divided into two categories: whether their husbands lived with them or stayed elsewhere. The wealth status of the respondents was categorized as poor, medium, and rich. The education status of the respondents was classified as no education, primary, secondary and higher level of education. The number of children ever born was grouped as no children, 1 to 2 children, three and more than three children. The fertility preference of the respondents was categorized as wanted more children, not sure about children, and wanted no more children. The numbers of dead children were classified as no dead children, one dead child, two, and more than two dead children. The variable named heard related to family planning strategies (FP) on the radiotelegraph, $\mathrm{TV}$, or in the newspaper was categorized as yes and no.

\subsection{Multinomial logistic regression}

This study uses Pearson's $\chi 2$ to evaluate the link between the outcome variable and the independent variables. To determine the affecting variables by using Determinants of Unintended Pregnancies, we use multinomial logistic regression. ${ }^{18}$ Logistic regression produces the coefficients (and its principle errors and necessary levels) of a formula to estimate the logit transformation of probability (p) of the existence of the trait of interest:

$\operatorname{logit}(p)=b_{0}+b_{1} X_{1}+b_{2} X_{2}+\ldots+b_{k} X_{k}$

where $p$ is the probability of the existence of the trait of interest. The logit transformation is defined as the logged odds:

Odds $=\frac{\mathrm{p}}{1-\mathrm{p}}=\frac{\text { Probality of presence of characteristic }}{\text { Probality of absence of charecteristic }}$

And

$\operatorname{logit}(\mathrm{p})=\log \log \left(\frac{\mathrm{p}}{1-\mathrm{p}}\right)$

Instead of selecting variables that reduce the grand total of squared errors (as in ordinary regression), the approximation in logistic regression determines variables that increase the chances of noticing the values of the sample.

\section{Results}

Table 1, briefly illustrates the facts and figures of the population on which the analysis was performed. Most of the study population (42.4\%) was in the age group $20-24$. The minimum population of $11.3 \%$ was in the age group 15-19. The large surveyed population was in the countryside $(75.9 \%$ ), while only $24.1 \%$ of the citizens were living in the city. Most of the women (69.4\%) reported that they got married after the age of 18 years. $89.1 \%$ of the women said that they are living with their husbands. Nearly half of the study population (47.2\%) was categorized as inferior. Women with high school (9-12) and vocational education were $61.7 \%$, whereas the women with only class $1-5$ education were only $13.2 \%$. Nearly half of the study population (47.0\%) had 1-2 children. On the other hand, only $14.1 \%$ of the study population had more than three children. $51.8 \%$ of the women wanted more children, whereas $10.1 \%$ were undecided on fertility preference. Most of the 
Table 1

Social and economical traits of present pregnant females in India.

\begin{tabular}{|c|c|c|}
\hline Current pregnancy intention & Frequency & Percent \\
\hline Wanted & 28386 & 88.1 \\
\hline Mistimed & 2256 & 7.0 \\
\hline Not wanted & 1582 & 4.9 \\
\hline Total & 32224 & 100.0 \\
\hline \multicolumn{3}{|l|}{ Age of respondent (years) } \\
\hline $15-19$ & 3632 & 11.3 \\
\hline $20-24$ & 13655 & 42.4 \\
\hline $25-29$ & 9770 & 30.3 \\
\hline $30+$ & 5167 & 16.0 \\
\hline Total & 32224 & 100.0 \\
\hline \multicolumn{3}{|l|}{ Type of residence } \\
\hline Urban & 7753 & 24.1 \\
\hline Rural & 24471 & 75.9 \\
\hline Total & 32224 & 100.0 \\
\hline \multicolumn{3}{|l|}{ Age at marriage (years) } \\
\hline$<18$ & 9876 & 30.6 \\
\hline$\geq 18$ & 22348 & 69.4 \\
\hline Total & 32224 & 100.0 \\
\hline \multicolumn{3}{|l|}{ Husband residence } \\
\hline Living with her & 28727 & 89.1 \\
\hline Staying elsewhere & 3497 & 10.9 \\
\hline Total & 32224 & 100.0 \\
\hline \multicolumn{3}{|l|}{ Wealth status } \\
\hline Poor & 15209 & 47.2 \\
\hline Medium & 6545 & 20.3 \\
\hline Rich & 10470 & 32.5 \\
\hline Total & 32224 & 100.0 \\
\hline \multicolumn{3}{|l|}{ Education } \\
\hline No education & 8100 & 25.1 \\
\hline Primary & 4253 & 13.2 \\
\hline Secondary + & 19871 & 61.7 \\
\hline Total & 32224 & 100.0 \\
\hline \multicolumn{3}{|l|}{ Offsprings ever born } \\
\hline Zero & 12533 & 38.9 \\
\hline One -two & 15153 & 47.0 \\
\hline Three + & 4538 & 14.1 \\
\hline Total & 32224 & 100.0 \\
\hline \multicolumn{3}{|l|}{ Fertility proclivity } \\
\hline Desired more & 16704 & 51.8 \\
\hline Unsettled & 3256 & 10.1 \\
\hline Desired no more & 12264 & 38.1 \\
\hline Total & 32224 & 100.0 \\
\hline \multicolumn{3}{|l|}{ Mortality of children } \\
\hline No death & 29216 & 90.7 \\
\hline One & 2447 & 7.6 \\
\hline Two + & 561 & 1.7 \\
\hline Total & 32224 & 100.0 \\
\hline \multicolumn{3}{|l|}{ Heard $F^{a}{ }^{a}$ on radio } \\
\hline No & 27106 & 84.1 \\
\hline Yes & 5118 & 15.9 \\
\hline Total & 32224 & 100.0 \\
\hline \multicolumn{3}{|l|}{ Heard FP ${ }^{\mathrm{a}}$ on Television } \\
\hline No & 15086 & 46.8 \\
\hline Yes & 17138 & 53.2 \\
\hline Total & 32224 & 100.0 \\
\hline \multicolumn{3}{|l|}{ Heard $\mathrm{FP}^{\mathrm{a}}$ in newspaper } \\
\hline No & 21984 & 68.2 \\
\hline Yes & 10240 & 31.8 \\
\hline Total & 32224 & 100.0 \\
\hline
\end{tabular}

${ }^{\mathrm{a}} \mathrm{FP}=$ Family Planning.

women did not listen to FP on radiotelegraph or newspaper. Almost half of the women (53.2\%) heard about FP on television. (88.1\%) women from the examined population illustrate that their pregnancy was desired, whereas only $4.9 \%$ of the women demonstrate that their pregnancy was undesired.

\subsection{Bivariate analysis}

From Table 2, it is demonstrated that countryside women have more chances to have an undesired pregnancy $(5.2 \%)$ as compared to that of the women of the city (4.1\%). The same sequence was noticed with ill-
Table 2

Pre-planning of pregnancy by choosing attributes of background.

\begin{tabular}{|c|c|c|c|c|c|}
\hline \multirow{2}{*}{$\begin{array}{l}\text { Background } \\
\text { features }\end{array}$} & \multicolumn{3}{|c|}{ Pregnancy intention } & \multirow{2}{*}{$\begin{array}{l}\text { Chi-square } \\
\text { value }\end{array}$} & \multirow{2}{*}{$\begin{array}{l}\mathrm{P}- \\
\text { Value }\end{array}$} \\
\hline & $\begin{array}{l}\text { Desired } \\
(\%)\end{array}$ & $\begin{array}{l}\text { Ill- } \\
\text { timmed } \\
(\%)\end{array}$ & $\begin{array}{l}\text { Undesired } \\
(\%)\end{array}$ & & \\
\hline \multicolumn{4}{|c|}{ Age of respondent } & 1042.981 & 0.000 \\
\hline $15-19$ & $\begin{array}{l}3288 \\
(90.5)\end{array}$ & $295(8.1)$ & $49(1.3)$ & & \\
\hline $20-24$ & $\begin{array}{l}12167 \\
(89.1)\end{array}$ & $\begin{array}{l}1174 \\
(8.6)\end{array}$ & $314(2.3)$ & & \\
\hline $25-29$ & $\begin{array}{l}8587 \\
(87.9)\end{array}$ & $599(6.1)$ & $584(6.0)$ & & \\
\hline $30+$ & $\begin{array}{l}4344 \\
(84.1)\end{array}$ & $188(3.6)$ & $635(12.3)$ & & \\
\hline \multicolumn{4}{|c|}{ Type of residence } & 41.669 & 0.000 \\
\hline Urban & $\begin{array}{l}6990 \\
(90.2)\end{array}$ & $447(5.8)$ & $316(4.1)$ & & \\
\hline Rural & $\begin{array}{l}21396 \\
(87.4)\end{array}$ & $\begin{array}{l}1809 \\
(7.4)\end{array}$ & $1266(5.2)$ & & \\
\hline \multicolumn{4}{|l|}{ Age at marriage } & 213.720 & 0.000 \\
\hline$<18$ & $\begin{array}{l}8444 \\
(85.5)\end{array}$ & $686(6.9)$ & $746(7.6)$ & & \\
\hline$\geq 18$ & $\begin{array}{l}19942 \\
(89.2)\end{array}$ & $\begin{array}{l}1570 \\
(7.0)\end{array}$ & $836(3.7)$ & & \\
\hline \multicolumn{4}{|c|}{ Husband residence } & 31.015 & 0.000 \\
\hline $\begin{array}{l}\text { Living with } \\
\text { her }\end{array}$ & $\begin{array}{l}25329 \\
(88.2)\end{array}$ & $\begin{array}{l}1946 \\
(6.8)\end{array}$ & $1452(5.1)$ & & \\
\hline $\begin{array}{l}\text { Staying } \\
\text { elsewhere }\end{array}$ & $\begin{array}{l}3057 \\
(87.4)\end{array}$ & $310(8.9)$ & $130(3.7)$ & & \\
\hline \multicolumn{4}{|l|}{ Wealth status } & 292.933 & 0.000 \\
\hline Poor & $\begin{array}{l}13018 \\
(85.6)\end{array}$ & $\begin{array}{l}1132 \\
(7.4)\end{array}$ & $1059(7.0)$ & & \\
\hline Medium & $\begin{array}{l}5829 \\
(89.1)\end{array}$ & $470(7.2)$ & $246(3.8)$ & & \\
\hline Rich & $\begin{array}{l}9539 \\
(91.1)\end{array}$ & $654(6.2)$ & $277(2.6)$ & & \\
\hline \multicolumn{4}{|l|}{ Education } & 560.948 & 0.000 \\
\hline No education & $\begin{array}{l}6820 \\
(84.2)\end{array}$ & $513(6.3)$ & 767 (9.5) & & \\
\hline Primary & $\begin{array}{l}3670 \\
(86.3)\end{array}$ & $330(7.8)$ & $253(5.9)$ & & \\
\hline Secondary + & $\begin{array}{l}17896 \\
(90.1)\end{array}$ & $\begin{array}{l}1413 \\
(7.1)\end{array}$ & $562(2.8)$ & & \\
\hline \multicolumn{4}{|c|}{ Children ever born } & 2972.095 & 0.000 \\
\hline 0 & $\begin{array}{l}11892 \\
(94.9)\end{array}$ & $565(4.5)$ & $76(0.6)$ & & \\
\hline $1-2$ & $\begin{array}{l}13153 \\
(86.8)\end{array}$ & $\begin{array}{l}1397 \\
(9.2)\end{array}$ & $603(4.0)$ & & \\
\hline $3+$ & $\begin{array}{l}3341 \\
(73.6)\end{array}$ & $294(6.5)$ & 903 (19.9) & & \\
\hline \multicolumn{4}{|c|}{ Fertility preference } & 1936.140 & 0.000 \\
\hline Wants more & $\begin{array}{l}15676 \\
(93.8)\end{array}$ & $873(5.2)$ & $155(0.9)$ & & \\
\hline Undecided & $\begin{array}{l}2987 \\
(91.7)\end{array}$ & $207(6.4)$ & $62(1.9)$ & & \\
\hline Wants no more & $\begin{array}{l}9723 \\
(79.3)\end{array}$ & $\begin{array}{l}1176 \\
(9.6)\end{array}$ & $1365(11.1)$ & & \\
\hline \multicolumn{4}{|l|}{ Children dead } & 174.671 & 0.000 \\
\hline No dead & $\begin{array}{l}25859 \\
(88.5)\end{array}$ & $\begin{array}{l}2067 \\
(7.1)\end{array}$ & $1290(4.4)$ & & \\
\hline 1 & $\begin{array}{l}2061 \\
(84.2)\end{array}$ & $162(6.6)$ & $224(9.2)$ & & \\
\hline $2+$ & $\begin{array}{l}466 \\
(83.1)\end{array}$ & $27(4.8)$ & $68(12.1)$ & & \\
\hline \multicolumn{4}{|c|}{ Heard $\mathbf{F P}^{\mathrm{a}}$ on radio } & 15.702 & 0.000 \\
\hline No & $\begin{array}{l}23803 \\
(87.8)\end{array}$ & $\begin{array}{l}1921 \\
(7.1)\end{array}$ & $1382(5.1)$ & & \\
\hline Yes & $\begin{array}{l}4583 \\
(89.5)\end{array}$ & $335(6.5)$ & $200(3.9)$ & & \\
\hline \multicolumn{4}{|c|}{ Heard FP $^{\mathrm{a}}$ on TV } & 177.637 & 0.000 \\
\hline No & $\begin{array}{l}12963 \\
(85.9)\end{array}$ & $\begin{array}{l}1139 \\
(7.6)\end{array}$ & $984(6.5)$ & & \\
\hline Yes & $\begin{array}{l}15423 \\
(90.0)\end{array}$ & $\begin{array}{l}1117 \\
(6.5)\end{array}$ & $598(3.5)$ & & \\
\hline \multicolumn{3}{|c|}{ Heard FP ${ }^{a}$ in newspaper } & & 196.540 & 0.000 \\
\hline \multicolumn{3}{|c|}{ No } & 1304 (5.9) & & \\
\hline
\end{tabular}


Table 2 (continued)

\begin{tabular}{|c|c|c|c|c|c|}
\hline \multirow{2}{*}{$\begin{array}{l}\text { Background } \\
\text { features }\end{array}$} & \multicolumn{3}{|c|}{ Pregnancy intention } & \multirow{2}{*}{$\begin{array}{l}\text { Chi-square } \\
\text { value }\end{array}$} & \multirow{2}{*}{$\begin{array}{l}\text { P- } \\
\text { Value }\end{array}$} \\
\hline & $\begin{array}{l}\text { Desired } \\
(\%)\end{array}$ & $\begin{array}{l}\text { Ill- } \\
\text { timmed } \\
(\%)\end{array}$ & $\begin{array}{l}\text { Undesired } \\
(\%)\end{array}$ & & \\
\hline & $\begin{array}{l}19020 \\
(86.5)\end{array}$ & $\begin{array}{l}1660 \\
(7.6)\end{array}$ & & & \\
\hline Yes & $\begin{array}{l}9366 \\
(91.5)\end{array}$ & $596(5.8)$ & $278(2.7)$ & & \\
\hline
\end{tabular}

${ }^{\text {a }} \mathrm{FP}=$ Family Planning.

timed pregnancy. Countryside women were more anticipated to have an ill-time pregnancy $(7.4 \%)$ as compared to that of the women of the city $(5.8 \%)$. Moreover, women that have got married at a young age (before 18 years) had a predominantly higher percentage of undesired pregnancy $(7.6 \%)$ as compared to the women that have got married at the age of 18 years or later $(3.7 \%)$.

Women who lived with their husbands were with a high unwanted pregnancy (5.1\%) than women who lived elsewhere. By concerning the socio-economic status, and consequences it is illustrated that females residing in comparatively penniless families mainly were expected to announce their pregnancy as undesirable (7.0\%), whereas those living in the privileged families were least anticipated to do so (2.6\%). Similarly, females living in relatively poor families mainly were expected to announce their pregnancies as ill-time (7.4\%), while those in the privileged families were least anticipated to do so $(6.2 \%)$.

Women with no schooling had the highest probability of undesired pregnancies $(9.5 \%)$, while the women with primary (class 1-5) school education had $(5.9 \%)$ of undesired pregnancies. Ill-time pregnancies have the highest probability $(7.8 \%)$ among women with the education of class $1-5$, and women (6.3\%) possibility od unintended pregnancies were in illiterate women.

Women that were having more than three children had the highest rate of undesired pregnancies (19.9\%) succeeding by females with 1-2 children (4.0\%) and the lowest among women with no children (0.6\%). Mistimed pregnancies were highest among females with one to two children (9.4\%), followed by women with more than three children (6.4\%), and lowest among women with no children (4.4\%).

Both undesired and ill-time pregnancies have higher rates among females who were illiterate about FP as compared to those who have knowledge about FP prior to their most current pregnancy.

After analyzing the above model in Table 3, it was found that the probability of mistimed pregnancy for women of age between 15 and 19 years was $7.896(6.246,9.981)$ times more than the women whose age was 30 years and older. The probability of mistimed pregnancy for women with age between 20 and 24 years. The age between 25 and 29 years was respectively having $4.134(3.460,4.940)$ and 2.008 (1.687, 2.390 ) times more probability of pregnancies than those of 30 years and older.

The females living in urban areas were $0.877(0.778,0.988)$ times less anticipated to have ill-time pregnancy than those living in rural areas.

The probability of mistimed pregnancy for the women who get married at a young age (before 18 years old) was $0.583(0.524,0.650)$ times less than the women who got married at the age of 18 and over. The results of this study showed that the probability of mistimed pregnancy of the women living with their husbands was $0.762(0.670,0.867)$ times less than women living elsewhere. Poor women had $0.980(0.862$, 1.113) times less probability of ill-time pregnancy than rich women. Similarly, women with medium wealth had $0.956(0.837,1.091)$ times less likelihood of mistimed pregnancy than rich women.

The probability of mistimed pregnancy for the women with no education was $0.773(0.684,0.873)$ times less than women with above secondary level of education. The likelihood of mistimed pregnancy for the women with no children was $0.217(0.176,0.268)$ times less than
Table 3

Illustrating various socio-economic and demographic factors influencing the Unintended Pregnancies in India by using Multinomial Logistic Regression.

\begin{tabular}{|c|c|c|c|c|c|c|}
\hline \multicolumn{2}{|c|}{ Current pregnancy intention } & \multirow[t]{2}{*}{$\beta$} & \multirow[t]{2}{*}{$\begin{array}{l}\text { P- } \\
\text { Value }\end{array}$} & \multirow[t]{2}{*}{ OR } & \multicolumn{2}{|c|}{$\begin{array}{l}95 \% \text { Confidence } \\
\text { Interval for OR }\end{array}$} \\
\hline & & & & & $\begin{array}{l}\text { Lower } \\
\text { Bounds }\end{array}$ & $\begin{array}{l}\text { Upper } \\
\text { Bounds }\end{array}$ \\
\hline \multirow[t]{43}{*}{ Mistimed } & Intercept & -2.642 & 0.000 & & & \\
\hline & \multicolumn{6}{|c|}{ Age of respondent } \\
\hline & $15-19$ & 2.066 & 0.000 & 7.896 & 6.246 & 9.981 \\
\hline & $20-24$ & 1.419 & 0.000 & 4.134 & 3.460 & 4.940 \\
\hline & $25-29$ & 0.697 & 0.000 & 2.008 & 1.687 & 2.390 \\
\hline & $\begin{array}{l}30+ \\
\text { (Reference) }\end{array}$ & $0^{\mathrm{b}}$ & . & . & . & . \\
\hline & \multicolumn{6}{|c|}{ Type of residence } \\
\hline & Urban & -0.132 & 0.031 & 0.877 & 0.778 & 0.988 \\
\hline & $\begin{array}{l}\text { Rural } \\
\text { (Reference) }\end{array}$ & $0^{\mathrm{b}}$ & . & . & . & . \\
\hline & \multicolumn{6}{|c|}{ Age at marriage } \\
\hline & $<18$ & -0.539 & 0.000 & 0.583 & 0.524 & 0.650 \\
\hline & $\geq 18$ & $0^{\mathrm{b}}$ & . & . & . & . \\
\hline & \multicolumn{6}{|c|}{ Husband residence } \\
\hline & $\begin{array}{l}\text { Living with } \\
\text { her }\end{array}$ & -0.272 & 0.000 & 0.762 & 0.670 & 0.867 \\
\hline & $\begin{array}{l}\text { Staying } \\
\text { elsewhere } \\
\text { Wealth status }\end{array}$ & $0^{\mathrm{b}}$ & . & . & . & . \\
\hline & Poor & -0.021 & 0.751 & 0.980 & 0.862 & 1.113 \\
\hline & Medium & -0.045 & 0.502 & 0.956 & 0.837 & 1.091 \\
\hline & Rich & $0^{\mathrm{b}}$ & . & . & . & . \\
\hline & \multicolumn{6}{|l|}{ Education } \\
\hline & No education & -0.258 & 0.000 & 0.773 & 0.684 & 0.873 \\
\hline & Primary & -0.065 & 0.340 & 0.937 & 0.820 & 1.071 \\
\hline & Secondary+ & $0^{\mathrm{b}}$ & . & . & . & . \\
\hline & \multicolumn{6}{|c|}{ Children ever born } \\
\hline & 0 & -1.527 & 0.000 & 0.217 & 0.176 & 0.268 \\
\hline & $1-2$ & -0.499 & 0.000 & 0.607 & 0.517 & 0.712 \\
\hline & $3+$ & $0^{\mathrm{b}}$ & . & . & . & . \\
\hline & \multicolumn{6}{|c|}{ Fertility preference } \\
\hline & Wants more & -0.607 & 0.000 & 0.545 & 0.490 & 0.606 \\
\hline & Undecided & -0.448 & 0.000 & 0.639 & 0.546 & 0.748 \\
\hline & $\begin{array}{l}\text { Wants no } \\
\text { more }\end{array}$ & $0^{\mathrm{b}}$ & . & . & . & . \\
\hline & \multicolumn{6}{|l|}{ Children dead } \\
\hline & No dead & 0.482 & 0.021 & 1.619 & 1.075 & 2.438 \\
\hline & 1 & 0.182 & 0.405 & 1.200 & 0.781 & 1.843 \\
\hline & $2+$ & $0^{\mathrm{b}}$ & . & . & . & . \\
\hline & Listen $\mathbf{F P}^{\star}$ on & adio & & & & \\
\hline & No & -0.061 & 0.350 & 0.940 & 0.827 & 1.070 \\
\hline & Yes & $0^{\mathrm{b}}$ & . & . & . & . \\
\hline & Listen $\mathbf{F P}^{*}$ on & & & & & \\
\hline & No & 0.091 & 0.090 & 1.095 & 0.986 & 1.216 \\
\hline & Yes & $0^{\mathrm{b}}$ & . & . & . & . \\
\hline & Listen $\mathbf{F P}$ in & ewspape & & & & \\
\hline & No & 0.232 & 0.000 & 1.262 & 1.118 & 1.423 \\
\hline & Yes & $0^{\mathrm{b}}$ & . & . & . & . \\
\hline Unwanted & Intercept & -2.342 & 0.000 & & & \\
\hline & Age of respon & ent & & & & \\
\hline & $15-19$ & -0.081 & 0.654 & 0.922 & 0.646 & 1.315 \\
\hline & $20-24$ & -0.462 & 0.000 & 0.630 & 0.530 & 0.750 \\
\hline & $25-29$ & -0.243 & 0.000 & 0.784 & 0.687 & 0.895 \\
\hline & $\begin{array}{l}30+ \\
\text { (Reference) }\end{array}$ & $0^{\mathrm{b}}$ & . & . & . & . \\
\hline & Type of reside & & & & & \\
\hline & Urban & 0.239 & 0.002 & 1.270 & 1.089 & 1.481 \\
\hline & $\begin{array}{l}\text { Rural } \\
\text { (Reference) }\end{array}$ & $0^{\mathrm{b}}$ & . & . & . & . \\
\hline & Age at marria & & & & & \\
\hline & $<18$ & 0.167 & 0.007 & 1.182 & 1.048 & 1.333 \\
\hline & $\geq 18$ & $0^{\mathrm{b}}$ & . & . & . & . \\
\hline & Husband resic & ence & & & & \\
\hline & $\begin{array}{l}\text { Living with } \\
\text { her }\end{array}$ & 0.291 & 0.004 & 1.338 & 1.099 & 1.628 \\
\hline & $\begin{array}{l}\text { Staying } \\
\text { elsewhere }\end{array}$ & $0^{\mathrm{b}}$ & . & . & . & . \\
\hline & Wealth status & & & & & \\
\hline & Poor & 0.519 & 0.000 & 1.680 & 1.397 & 2.020 \\
\hline
\end{tabular}


Table 3 (continued)

\begin{tabular}{|c|c|c|c|c|c|}
\hline \multirow[t]{2}{*}{ Current pregnancy intention } & \multirow[t]{2}{*}{$\beta$} & \multirow[t]{2}{*}{$\begin{array}{l}\text { P- } \\
\text { Value }\end{array}$} & \multirow[t]{2}{*}{ OR } & \multicolumn{2}{|c|}{$\begin{array}{l}\text { 95\% Confidence } \\
\text { Interval for OR }\end{array}$} \\
\hline & & & & $\begin{array}{l}\text { Lower } \\
\text { Bounds }\end{array}$ & $\begin{array}{l}\text { Upper } \\
\text { Bounds }\end{array}$ \\
\hline Medium & 0.237 & 0.018 & 1.267 & 1.042 & 1.541 \\
\hline $\begin{array}{l}\text { Rich } \\
\text { Education }\end{array}$ & \multicolumn{4}{|c|}{ Education } & . \\
\hline No education & 0.138 & 0.065 & 1.148 & 0.991 & 1.330 \\
\hline Primary & 0.054 & 0.539 & 1.056 & 0.887 & 1.257 \\
\hline Secondary + & $0^{\mathrm{b}}$ & . & . & . & . \\
\hline \multicolumn{6}{|c|}{ Children ever born } \\
\hline 0 & -2.339 & 0.000 & 0.096 & 0.071 & 0.131 \\
\hline $1-2$ & -1.416 & 0.000 & 0.243 & 0.210 & 0.280 \\
\hline $3+$ & $0^{\mathrm{b}}$ & . & . & . & . \\
\hline \multicolumn{6}{|c|}{ Fertility preference } \\
\hline Wants more & -1.883 & 0.000 & 0.152 & 0.127 & 0.183 \\
\hline Undecided & -1.634 & 0.000 & 0.195 & 0.150 & 0.254 \\
\hline $\begin{array}{l}\text { Wants no } \\
\text { more }\end{array}$ & $0^{\mathrm{b}}$ & . & . & . & . \\
\hline \multicolumn{6}{|l|}{ Children dead } \\
\hline No dead & 0.711 & 0.000 & 2.035 & 1.539 & 2.691 \\
\hline 1 & 0.308 & 0.046 & 1.361 & 1.005 & 1.843 \\
\hline $2+$ & $0^{\mathrm{b}}$ & . & . & . & . \\
\hline \multicolumn{6}{|c|}{ Listen FP on radio } \\
\hline No & -0.023 & 0.788 & 0.977 & 0.824 & 1.158 \\
\hline Yes & $0^{\mathrm{b}}$ & . & . & . & . \\
\hline \multicolumn{6}{|c|}{ Listen FP on TV } \\
\hline No & 0.095 & 0.171 & 1.099 & 0.960 & 1.259 \\
\hline Yes & $0^{\mathrm{b}}$ & . & . & . & . \\
\hline \multicolumn{6}{|c|}{ Listen FP in the newspaper } \\
\hline No & 0.059 & 0.505 & 1.061 & 0.892 & 1.262 \\
\hline Yes & $0^{\mathrm{b}}$ & . & . & . & . \\
\hline
\end{tabular}

a The reference group is: Wanted.

b This variable is set to 0 because it is unnecessary.

* FP = Family Planning.

women with more than three children. The likelihood of mistimed pregnancy for the women who wanted more children was $0.545(0.490$, 0.606 ) times less than women who wanted no more children.

Women with no children's deaths had 1.619 (1.075, 2.438) times more anticipated announcing mistimed pregnancy than the women with more than two dead children. On the other hand, women with one dead child had $1.200(0.781,1.843)$ times more probability of mistimed pregnancy than women with more than two dead children.

\subsection{Correlates of unwanted pregnancy}

It was found that the probability of unwanted pregnancy for women age $15-19$ years was $0.922(0.646,1.315)$ times less than the women whose age is 30 years and older. The probability of unwanted pregnancy for the women whose age is between 20 to 24 years and 25-29 years were respectively $0.630(0.530,0.750)$ and $0.784(0.687,0.895)$ times less than the women with age of 30 years and over.

The probability of unwanted pregnancy as compared to wanted pregnancy for the women living in urban areas was $1.270(1.089,1.481)$ times more than women living in rural areas. The probability of unwanted pregnancy as compared to wanted pregnancy for the women who got married before 18 years old was $1.182(1.048,1.333)$ times more than women who got married aged 18 and over.

The probability of unwanted pregnancy compared to wanted pregnancy for the women living with their husband was $1.338(1.099,1.628)$ times more than women living elsewhere.

Poor women had $1.680(1.397,2.020)$ times more probability of undesired pregnancy as compared to desired pregnancy than rich women.

The probability of undesired pregnancy in comparison to desired pregnancy for the women with no education was $1.148(0.991,1.330)$ times more than women with an above secondary level of education.

The probability of unwanted pregnancy for women with no children was $0.096(0.071,0.131)$ times less as compared to the women with more than three children. Women with $1-2$ children had $0.243(0.210$, 0.280 ) times less probability of undesired pregnancy as compared to desired pregnancy than women with more than three children.

The probability of undesired pregnancy in comparison to desired pregnancy for the women who wanted more children was $0.152(0.127$, 0.183) times less than women who wished no more children. The probability of undesired pregnancy as compared to desired pregnancy for the women undecided about children was $0.195(0.150,0.254)$ times less than women who wanted no more children. Women with no dead offspring had $2.035(1.539,2.691)$ times more probability of unwanted pregnancy than women with greater than two dead children. Women with one dead child had $1.361(1.005,1.843)$ times more likelihood of unwanted pregnancy than women with more than two dead children.

\section{Discussion}

To determine the triggering factors of unwanted pregnancy in India was the main focus of this study, revealing that undesired pregnancy is widespread among Indian females. The bivariate analysis showed that determinants such as age, type of residence, age group at the first wedding, husband residence, wealth status, education, total offsprings ever born, fertility preference, subtotal of dead children, and exposure of radio, television, and newspaper were significantly associated with an unintended pregnancy. In the multivariate statistical analysis, the age group of females, type of residence, age group at the first wedding, husband residence, wealth status, children ever born, fertility preference, heard Family Planning on TV and newspaper were presented to affect undesired pregnancy most significantly. This review has illustrated that the women of the younger age group have a higher chance of having the recent pregnancy as an ill-time one. Mistimed pregnancy is higher among younger women of the teenage group and decreases with the rising age of the women. These findings are similar to the studies reported by. ${ }^{19-22}$ Respondents with the age of more than 30 are mostly expected to face an undesired pregnancy. A similar result was found for married pregnant women in Indonesia, which shows that higher the age groups from which women belong, the higher the probability of having unwanted pregnancies will beThe survey organized in Egypt also confirmed the same result ${ }^{23}$ (Jaeni et al., 2007). Analysis of this study revealed that women living in urban areas had fewer chances to have ill time pregnancies than those residing in rural areas. ${ }^{24}$ This could be due to poverty, limited availability of health services, and poor utilization of health care facilities, and ignorance. ${ }^{25}$ We have found a significant relationship among age at first marriage with mistimed and not wanted pregnancy in India. Respondents that belong to the age group of fewer than 18 years were more anticipated to have not desired pregnancies than the women with age of more than or equal 18 years. Similar findings have been reported in Ethiopia. ${ }^{26,27}$ The reasons behind this could be that the women who were married before 18 years are not prepared psychologically, economically, and physically to have pregnancy. ${ }^{27,28}$ Women 's economic status has an important influence on unwanted pregnancy, and the results of this are similar to the finding of. ${ }^{27}$ Women from poor family backgrounds were more anticipated to have unwanted pregnancies than the women with wanted pregnancies as compared to the women from wealthy family backgrounds. ${ }^{28}$ The current study revealed that there is no significant relationship between respondent educations with the incident of unwanted pregnancy. ${ }^{29-31}$ It has been reported the same findings were seen in Japan, Kenya, and Nepal, respectively. The current study's results contradict the usual typical perception and other articles that show the association between unwanted pregnancy and women's education. ${ }^{28,32}$ Women with a desire to have more children but were unsettled were less anticipated to announce unintended pregnancy than those who want no more children. The same findings have been reported in Africa by. ${ }^{19,23}$ A respondent with 0 or $1-2$ children is less likely to have mistimed pregnancy as compared to the respondent with three or more children. This is because 
the respondent that has no or $1-2$ children wants more children; consequently, they want to be pregnant. ${ }^{23}$ The multivariate analysis consequences illustrate that those who have no daily approach to mass communication (Radio, TV, and Newspaper) were more anticipated to announce mistimed pregnancy as compared to those who have access to mass communication. It is signified that mass communication has an essential role in decreasing unwanted pregnancies because it gives a broader array of awareness. ${ }^{33,34}$

\section{Conclusion}

In conclusion, the high percentage of unwanted pregnancies is not caused by a single factor. Numerous variables have contributed to this respect. Amongst them, this review has revealed that the age group of females, type of residence, age at the first wedding, husband residence, wealth status, children ever born, fertility preference, heard FP on TV and newspaper are the most powerful determinants of unwanted pregnancies. In brief, the objective of the reproductive health plan is to remove unwanted pregnancies by concentrating on all these acknowledged determinants. Children and mother death, disease, and the necessity for abortion are declined, and the thorough health status of the family members is sustained and improved.

\section{Ethical approval}

Not applicable.

\section{Funding}

This research did not receive any specific grant from funding agencies in the public, commercial, or not-for-profit sectors.

\section{Consent to participate}

Not applicable.

\section{Availability of data and material}

Data are available upon request from DHS data.

\section{Declaration of competing interest}

The authors have no conflicts of interest to declare.

\section{Acknowledgment}

We want to show our appreciation to the Demographic and Health Surveys (DHS) Program for offering data access used in this research. The authors also declare the following issues.

\section{References}

1 Santelli J, Rochat R, Hatfield-Timajchy K, et al. The measurement and meaning of unintended pregnancy. Perspect Sex Reprod Health. 2003:94-101.

2 Henshaw SK. Unintended pregnancy in the United States. Fam Plann Perspect. 1998: $24-46$.

3 Chinebuah B, Pérez-Escamilla R. Unplanned pregnancies are associated with less likelihood of prolonged breast-feeding among primiparous women in Ghana. J Nutr. 2001;131:1247-1249.
4 Kamal M, Islam A. Prevalence and socioeconomic correlates of unintented pregnancy among women in rural Bangladesh. Salud Publica Mex. 2011;53:108-115.

5 Marston C, Cleland J. Do unintended pregnancies carried to term lead to adverse outcomes for mother and child? An assessment in five developing countries. Popul Stud. 2003;57:77-93.

6 Eggleston E. Unintended Pregnancy and Use of Prenatal Care in Ecuador. annual meeting of the American Public Health Association, Washington, DC, Nov. Vol vol. 151998:19.

7 Huda FA, Chowdhuri S, Robertson Y, et al. Understanding Unintended Pregnancy in Bangladesh: Country Profile Report. 2013.

8 Habitu YA, Yeshita HY, Dadi AF, Galcha D. Prevalence of and factors associated with emergency contraceptive use among female undergraduates in Arba Minch University, Southern Ethiopia, 2015: a cross-sectional study. Int. J. Popul. Res. 2018; 2018.

9 Islam MM, Rashid M. DETERMINANTS OF UNINTENDED fl PREGNANCY AMONG EVER-MARRIED WOMEN IN BANGLADESH. 2005.

10 Palamuleni ME, Adebowale AS. Prevalence and determinants of unintended pregnancies in Malawi. Afr Popul Stud. 2014;28:551-563.

11 Huynh ST, Yokomichi H, Akiyama Y, et al. Prevalence of and Factors Associated with Unplanned Pregnancy Among Women in Koshu, Japan: Cross-Sectional Evidence from Project Koshu, 2011-2016. vol. 20. 2020:1-10.

12 Erfani A, Hosseini H, Nojomi MJW, health. Unintended Pregnancies in Hamedan, Iran: Levels and Determinants. vol. 59. 2019:318-333.

13 Bastola K, Neupane S, Hadkhale K, Kinnunen TJJWH. Issues care 4. Unintended pregnancy among married pregnant women in Nepal. 2015;4:2.

14 Sukhini K, Sheela SJH. Knowledge, Attitude and Practise of Oral Contraceptive Pills Among Postnatal Mothers and Their Impact after Educating Them.vol. 87:83.87.

15 Kost K, Finer LB, Singh SJPos, health r. Variation in State Unintended Pregnancy Rates in the United States. vol. 44. 2012:57-64.

16 Horvath S, Schreiber CAJCpr. Unintended Pregnancy, Induced Abortion, and Mental Health. vol. 19. 2017:1-6.

17 IIPS I. National Family Health Survey (NFHS-4), 2015-16. Mumbai, India: International Institute for Population Sciences (IIPS); 2017.

18 Starkweather J, Moske AK. Multinomial logistic regression. Consulted page at September 10th. 29. 2011:2825-2830. http://www.unt.edu/rss/class/Jon/Benchmarks/ MLR_JDS_Aug2011.pdf.

19 Adetunji J. Levels, Trends and Determinants of Unintended Childbearing in Developing Countries. Washington, DC: annual meeting of the Population Association of America; 1997.

20 Abbasi-Shavazi MJ, Hosseini-Chavoshi M, Aghajanian A, Delavar B, Mehryar A. Un in tended Pregnancies in the Is lamic Re pub lic of Iran: lev els and Correlates. Asia Pac Popul J. 2004;19:27.

21 Najafian M, Karami K, Cheraghi M, Mohammad Jafari R. Prevalence of and some factors relating with unwanted pregnancy, in ahwaz city, Iran, 2010. ISRN obstetrics and gynecology. 2011:2011.

22 Geda NR, Lako TK. Unintended pregnancy among married women in Damot Gale District, Southern Ethiopia: examining the prevalence and risk factors. Afr Popul Stud. 2012;26.

23 Shaheen A, Diaaeldin M, Chaaya M, El Roueiheb Z. Unintended pregnancy in Egypt: evidence from the national study on women giving birth in 1999. EMHJ-Eastern Mediterranean Health Journal. 2007;13(6):1392-1404, 2007.

24 Lawani L, Ekem N, Eze J, Ekwedigwe K, Egede J, Isikhuemen M. The prevalence and determinants of unintended pregnancies among women in Abakaliki, Southeast Nigeria. Global J Health Sci. 2018;10:65-73.

25 Worku S, Fantahun M. Unintended pregnancy and induced abortion in a town with accessible family planning services: the case of Harar in eastern Ethiopia. Ethiop $J$ Health Dev. 2006;20:79-83.

26 Geda NR, Lako TK. A population based study on unintended pregnancy among married women in a district in Southern Ethiopia. J Geogr Reg Plann. 2011;4: $417-427$.

27 Nwokocha EE. Pregnancy Outcomes Among the Ibani of Rivers State, Nigeria: Findings from Case-Studies. 2006.

28 Finer LB, Henshaw SK. Disparities in rates of unintended pregnancy in the United States, 1994 and 2001. Perspect Sex Reprod Health. 2006;38:90-96.

29 Goto A, Yasumura S, Reich MR, Fukao A. Factors associated with unintended pregnancy in Yamagata, Japan. Soc Sci Med. 2002;54:1065-1079.

30 Kassa N, Berhane Y, Worku A. Predictors of unintended pregnancy in kersa, eastern Ethiopia, 2010. Reprod Health. 2012;9:1.

31 Habte D, Teklu S, Melese T, Magafu MG. Correlates of unintended pregnancy in Ethiopia: results from a national survey. PLoS One. 2013;8, e82987.

32 Finer LB, Zolna MR. Unintended pregnancy in the United States: incidence and disparities, 2006. Contraception. 2011;84:478-485.

33 Flora JA, Maibach EW. Cognitive responses to AIDS information: the effects of issue involvement and message appeal. Commun Res. 1990;17:759-774.

34 Oni GA, McCarthy J. Contraceptive knowledge and practices in Ilorin, Nigeria: 198388. Stud Fam Plann. 1990;21:104-109. 